\title{
Rapid, Sensitive and Validated Ultra-Performance Liquid Chromatography/Mass Spectrometric Method for the Determination of Fenofibric Acid and its Application to Human Pharmacokinetic Study
}

\author{
SUNIL K DUBEY*, MANOJ S. TOMAR, ANIL KUMAR PATNI, \\ ARSHAD KHUROO, SIMRIT REYAR and TAUSIF MONIF \\ Department of Clinical Pharmacology and Pharmacokinetics, \\ Ranbaxy Laboratories Ltd., Plot No. 20, Sector-18, \\ Udyog Vihar Industrial Area, Gurgaon, Haryana, India. \\ tausif.monif@ranbaxy.com
}

Received 26 March 2009; Accepted 20 May 2009

\begin{abstract}
The first, rapid and sensitive ultra performance liquid chromatography mass spectrometric method for the determination of fenofibric acid, the active metabolite of fenofibrate, a lipid regulating agent, in human EDTA plasma has been developed and validated using fenofibric d6 acid as internal standard and Waters LC-MS/MS. Negative ions of fenofibric acid and fenofibric d6 acid were detected in multiple reaction-monitoring (MRM) mode. The method was validated over a concentration range of $0.176 \mu \mathrm{g} / \mathrm{mL}$ to $19.837 \mu \mathrm{g} / \mathrm{mL}(\mathrm{r} \geq 0.99)$. It took only 1.5 minute to analyse a sample. Intraand inter-run precision of fenofibric acid assay at four concentrations ranged from $0.5 \%$ to $4.3 \%$ with accuracy varied from 93.1 to $108.1 \%$ indicating good precision and accuracy. Analytical recoveries of fenofibric acid and internal standard in plasma were less than $90 \%$. This method was successfully applied for evaluation of pharmacokinetics of fenofibric acid after a single oral dose of $145 \mathrm{mg}$ fenofibrate to 10 Indian healthy volunteers.
\end{abstract}

Keywords: Validation, LC-MS/MS, Fenofibric Acid, Pharmacokinetics, Lipid regulating agent.

\section{Introduction}

Fenofibric acid is chemically 2-[4'-( $p$-chlorobenzoyl)phenoxy]-2-methylpropionic acid. Fenofibrate is a lipid regulating agent. Fenofibric acid, the active metabolite of fenofibrate, produces reductions in total cholesterol, LDL cholesterol, apolipoprotein B, total triglycerides and triglyceride rich lipoprotein (VLDL) in treated patients. In addition, treatment with fenofibrate results in increase in high density lipoprotein (HDL) and apoproteins apoAI 
and apoAII. The effects of fenofibric acid seen in clinical practice have been explained in vivo in transgenic mice and in vitro in human hepatocyte cultures by the activation of peroxisome proliferator activated receptor $\alpha(\mathrm{PPAR} \alpha)$. Through this mechanism, fenofibrate increases lipolysis and elimination of triglyceride-rich particles from plasma by activating lipoprotein lipase and reducing production of apoprotein C-III (an inhibitor of lipoprotein lipase activity $)^{1-4}$. Previously fenofibric acid has been determined in human plasma simultaneously with other statins by LC-MS/MS ${ }^{5,6}$. The LC-MS/MS method described in this paper allow the lower limit of quantitation (LLOQ) of fenofibric acid in human plasma down to 0.176 $\mu \mathrm{g} / \mathrm{mL}$ which is quite low than used in previous methodologies ${ }^{5,6}$. The generic forms of fenofibrate were recently produced to meet the demand of the global healthcare market. To assist in regulatory filing, product quality control, and dose regimen design for clinical trials, characterization of human pharmacokinetics is generally the first and foremost clinical investigation of new drugs. Since the introduction of the electrospray ionization (ESI) and atmospheric pressure chemical ionization (APCI) techniques, LC-MS/MS has become an ideal and widely used method in characterization and quantitative analysis of drugs and their metabolites owing to its superior specificity, sensitivity and efficiency over others such as radioimmunoassay (RIA) gas chromatography/mass spectrometry (GC/MS) and liquid chromatography (LC) with UV. Buffer systems made from 2 to $5 \mathrm{mM}$ ammonium acetate provide sufficient repeatability of the retention times and efficient ionization of acidic compounds in negative ion ESI. Having said that, the aim of the present study was to develop a rapid and very sensitive LC-MS/MS method for quantitative determination of fenofibric acid in human biomatrices, and utilize the method, after its full validation, to characterize fenofibric acid pharmacokinetics in healthy human volunteers.

\section{Experimental}

Fenofibric acid was purchased from Varda Biotech (Mumbai, India). Fenofibric d6 acid was procured from CDN Isotopes, Canada. Acetonitrile was purchased from Spectrochem Pvt. Ltd. (Mumbai, India). Methanol was purchased from Qualigens fine chemicals (A division of GSK Ltd, Mumbai, India). Human plasma containing ethylene diamine tetra acetic acid (EDTA) as anticoagulant was collected in-house which were free from HIV and Hepatitis. All other solvents and reagents of analytical grade were purchased from S.d. fine chem. Ltd (Mumbai, India).

\section{Instrumentation and conditions}

Chromatographic separation was achieved by using the Acquity UPLC BEH C18 $(2.1 \times 50 \mathrm{~mm}, 1.7 \mu \mathrm{m})$ maintaining the column oven temperature at $35^{\circ} \mathrm{C}$. A mixture of acetonitrile, ammoniun acetate buffer $(70: 30 ; \mathrm{v} / \mathrm{v})$ was used as the mobile phase with flow rate of $0.25 \mathrm{~mL} / \mathrm{min}$. The samples were loaded in the UPLC auto-sampler and temperature of the auto sampler was set at $10^{\circ} \mathrm{C} .5 \mu \mathrm{L}$ of sample extract was injected and the eluent was monitored by tandem mass spectrometry with turbo ion spray ionization in negative ion mode of Waters Quattro premier mass spectrometer. The chromatographic data was acquired and processed using computer based Mass Lynx software Version 1.4.1. The negative ions were monitored in the multiple reaction monitoring (MRM) mode. The following ion transitions $(\mathrm{m} / \mathrm{z}$ ) were monitored $316.90 \mathrm{~m} / \mathrm{z}$ (parent) and 230.94 (product) for Fenofibric acid and 322.47 (parent) and 230.96 (Product) for fenofibric d6 acid with the capillary voltage set at $3 \mathrm{kV}$. The source and desolvation temperature was set at $100{ }^{\circ} \mathrm{C}$ and $350{ }^{\circ} \mathrm{C}$ respectively. Cone and desolvation gas flow were maintained at 40 and $1100 \mathrm{~L} / \mathrm{h}$ respectively. The argon gas collision induced dissociation was used with the energy $15 \mathrm{e} \mathrm{V}$. The total run time for an LC-MS/MS was 1.5 minutes. 


\section{Stock solution, Calibration curve $(C C)$ and Quality Control $(Q C)$ sample preparation}

Approximately $1000 \mu \mathrm{g} / \mathrm{mL}$ of stock solution for fenofibric acid was prepared using HPLC grade methanol. This solution was diluted to make a series of standard working solutions in the range from 8.819 to $991.869 \mu \mathrm{g} / \mathrm{mL}$. All the working solutions were freshly prepared and stored shaded from light at $1-10{ }^{\circ} \mathrm{C}$. Calibration standard and QC samples in plasma were prepared by spiking corresponding working solutions in the drug free human plasma. The final concentrations of calibration standard in plasma were 0.176 , $0.462,0.608,1.215,2.430,4.860,9.720,13.886$ and $19.837 \mu \mathrm{g} / \mathrm{mL}$, respectively. The final concentrations of QC in plasma were $0.177,0.472,6.941,13.882 \mu \mathrm{g} / \mathrm{mL}$, respectively. The plasma samples were stored below $-50{ }^{\circ} \mathrm{C}$ and for long-term stability samples were stored below $-15{ }^{\circ} \mathrm{C}$ as well as $-50{ }^{\circ} \mathrm{C}$.

\section{Sample preparation for plasma}

Aliquots of human plasma containing both analyte and its internal standard (fenofibric d6 acid) were extracted by solid phase extraction (HLB cartridges, $30 \mathrm{mg} / 1 \mathrm{cc}$ ) method. $50 \mu \mathrm{L}$ of internal standard stock dilution (fenofibric d6 acid, approximately $10.0 \mu \mathrm{g} / \mathrm{mL}$ ) and $500 \mu \mathrm{L}$ aliquot of each sample were added into polypropylene tubes. $200 \mu \mathrm{L}$ of HPLC grade water was added to it and vortexed. The cartridges were conditioned with methanol $(1 \mathrm{~mL})$ and equilibrated with de-ionized water $(1 \mathrm{~mL})$. The samples were loaded onto the cartridges and the cartridges were washed with $1 \mathrm{~mL}$ HPLC grade water and eluted the samples twice with $1 \mathrm{~mL}$ of methanol. The eluates were evaporated to dryness at $50{ }^{0} \mathrm{C}$ under Nitrogen gas pressure. Dried residues were then reconstituted with $400 \mu \mathrm{L}$ of mobile phase $(70: 30 \mathrm{v} / \mathrm{v}$ acetonitrile: ammonium acetate buffer).

\section{Assay characteristics for method validation}

A simple method for determination of fenofibric acid was developed and validated in terms of selectivity, sensitivity, linearity of response, accuracy, precision, recovery, stability, dilution integrity, effect of anticoagulant as well as matrix effect according to US-FDA guidelines for validation of bioanalytical methods ${ }^{7-11}$.

\section{Selectivity}

The selectivity of this method was investigated by analyzing six individual human blank plasma samples. Each blank sample was tested for interferences using the proposed extraction procedure and LC-MS/MS conditions.

\section{Sensitivity}

LLOQ was defined as the lowest concentration at which the precision, expressed as $\%$ coefficient of variance ( $\% \mathrm{CV}$ ), is less than $20 \%$ and the accuracy is within $\pm 20 \%$.

\section{Matrix effect}

Matrix effect is due to co-elution of some endogenous components present in biological samples. These components may not give a signal in MRM of target analyte, but can certainly decrease or increase the response of the analyte dramatically to affect the sensitivity, accuracy and precision of the method. Thus assessment of matrix effect constitutes an important and integral part of validation for quantitative LC-MS/MS method for supporting pharmacokinetic studies ${ }^{12}$. To evaluate the matrix effect, chromatographic peak areas of fenofibric acid from the spike-after preparation. samples were compared to the neat standards at the QC concentrations. 


\section{Linearity}

Biological samples were quantified using the ratio of peak area of fenofibric acid to that of internal standard. The calibration curves were established through a linear least-squares regression with a weighing factorof $1 / \mathrm{C}^{2}$, where $\mathrm{C}$ is the concentration of the calibration standards. Coefficients of correlations ( $r$ ) were required to be 0.99 or better. Concentration in the QC and unknown biological samples were quantified from the regression equation.

\section{Accuracy and precision}

The precision and accuracy of the method were assessed by intra- and inter-day validation. The intra- and inter-day accuracy and precision were determined by determining the concentrations of fenofibric acid in plasma in six replicates of QC samples for three separate batches. Precision was expressed as the \% CV. Accuracy was expressed as the percent deviation of the mean determined concentration against the spiked concentration. A \% CV and $\%$ accuracy less than or equal to a limit of $15 \%$ in measuring range above LLOQ and less than $20 \%$ at the level of LLOQ were demanded.

\section{The recovery}

The percentage recovery of fenofibric acid and internal standard was determined by measuring the mean peak area response of six replicates of extracted quality control samples (LQC, MQC and HQC) against the mean peak area response of six replicates of aqueous (unextracted) quality control samples at concentrations representing $100 \%$ extraction of quality control samples at low, middle and high concentration ${ }^{13}$.

\section{Stability}

Stability experiments were performed to evaluate stabilities of the analytes in plasma samples under different storage and processing conditions. The freeze-thaw stability of spiked plasma samples was determined after three freeze thaw cycles. Six replicate of low and high quality control samples were analysed after third freeze thaw cycle, each cycle consisted of removing the samples from the freezer, thawing them unassisted to room temperature kept at room temperature for $2 \mathrm{~h}$ and re-freezing at $-50{ }^{\circ} \mathrm{C}$. The percentage degradation was determined by calculating the concentration of stability quality control samples against freshly spiked calibration curve. Long-term stability: Long-term stability of fenofibric acid was evaluated using low and high QC samples stored below $-15{ }^{\circ} \mathrm{C}$ in cold room and below $-50{ }^{\circ} \mathrm{C}$ in deep freezer for a period of 102 days. Six replicates of low and high quality control samples were used for each stability exercise. The stored QC samples were analyzed against freshly spiked calibration curve.

In addition to this, bench top, in-injector, post preparative, stock solution and short-term stability for both fenofibric acid and fenofibric d6 acid were determined and are discussed in result and discussion section.

\section{Dilution integrity}

Dilution integrity was determined by spiking about 1.79 times upper limit of quantitation (ULOQ) concentration for fenofibric acid. This was then diluted by factor of 2 and 4 with drug free plasma. These samples were processed and analysed against freshly spiked calibration standards.

\section{Method application}

The established method was applied in a pharmacokinetic study performed in healthy Indian subjects. Ten healthy volunteers were enrolled in this study in Clinical Pharmacology Unit, 
Ranbaxy Laboratories Limited, Noida (India). The volunteers were free of cardiac, hepatic, renal, pulmonary, neurologic, gastrointestinal and hematologic disease, as assessed by physical examination, electrocardiography and the laboratory tests including hematology, biochemistry, electrolytes and urinalysis. All the subjects were instructed to abstain from taking any medication for 2 weeks before and during the whole study period. The study protocol was approved by the local Ethical Review Committee in accordance with the principles of the Declaration of Helsinki, and the recommendations of the Food and Drug Administration of United States (USFDA). Written informed consent was obtained from all subjects. Data analysis was carried out using the SAS system for Windows, release 9.1 (SAS Institute Inc.,USA) and the WinNonlin PK Software, Version 5.0.1. The study was designed as randomized, two-treatment, two-period, two-sequence, single-dose, crossover bioequivalence study. A single oral dose of fenofibrate $145 \mathrm{mg}$ of Ranbaxy Research Laboratories will be administered with $240 \mathrm{~mL}$ of drinking water at ambient temperature 30 minutes after serving of a high-fat high-calorie breakfast. Following an overnight fast of at least $10 \mathrm{~h}$, subjects will start the recommended meal 30 minutes prior to administration of the drug product Subjects will receive standardized meals - lunch, snacks and dinner at 4, 9 and $13 \mathrm{~h}$, respectively, after drug administration. Venous blood samples each about $5 \mathrm{~mL}$ were drawn in K3 EDTA vacutainers before dosing $(0 \mathrm{~h})$ and at $0.50,1.00,1.50,2.00,2.50,3.00,3.50$, $4.00,4.50,5.00,5.50,6.00,6.50,7.00,7.50,8.00,8.50,9.00,10.00,11.00,12.00,16.00,20.00$, $24.00,36.00,48.00,72.00$ and 96.00 hour post dose in each period. Plasma samples were separated with centrifugation at $1500 \mathrm{rpm}$ for 15 minutes immediately. All samples were stored below $-50 \circ \mathrm{C}$ until analysis ${ }^{14,15}$.

\section{Results and Discussion}

\section{Chromatographic and MS/MS conditions}

Method development began with the optimization of chromatographic conditions including mobile phase composition and column type. The feasibility of various mixtures of solvents such as methanol acetonitrile with buffers such as ammonium acetate, ammonium formate was tested. Mobile phase comprising of acetonitrile: ammonium acetate buffer (70:30; v/v) was found to be suitable for the LC separation with ESI mass detection of the analytes. Chromatographic separation was achieved by using the Acquity UPLC BEH C18 (2.1x $50 \mathrm{~mm}, 1.7 \mu \mathrm{m})$ maintaining the column oven temperature at $35^{\circ} \mathrm{C}$. Mass spectrometric conditions were optimized so as to achieve the maximum stable response of the parents and the major product ions of the analytes. Negative ions were monitored in the Multiple Reaction-Monitoring (MRM) mode. The following ion transitions $(\mathrm{m} / \mathrm{z}$ ) were monitored $316.90 \mathrm{~m} / \mathrm{z}$ (parent) and 230.94 (product) for Fenofibric acid and 322.47 (parent) and 230.96 (Product) for fenofibric d6 acid. The full-scan mass spectra of fenofibric acid and fenofibric d6 acid are shown in Figure 1(a) and 1(b), respectively. Chemical structures of fenofibric acid and fenofibric d6 acid are shown in Figure 2. Parameters such as capillary voltage, source and desolvation temperature, cone and desolvation gas flow and the collision gas pressure and energy were tuned to maximize the selectivity and sensitivity for the detection.

\section{Sample preparation}

SPE (solid phase extraction) is often preferred over liquid/liquid extraction because of the practical advantages of no emulsions, better recoveries, cleaner extracts achievable, the ability to remove many interferences and matrix components selectively, and so on. And what has become one of the most important advantages for SPE is that it is much more capable of being automated, which is important in reducing overall cycle times for clinical studies. Thus, a HLB cartridge (Waters) was tested for sample pretreatment. 


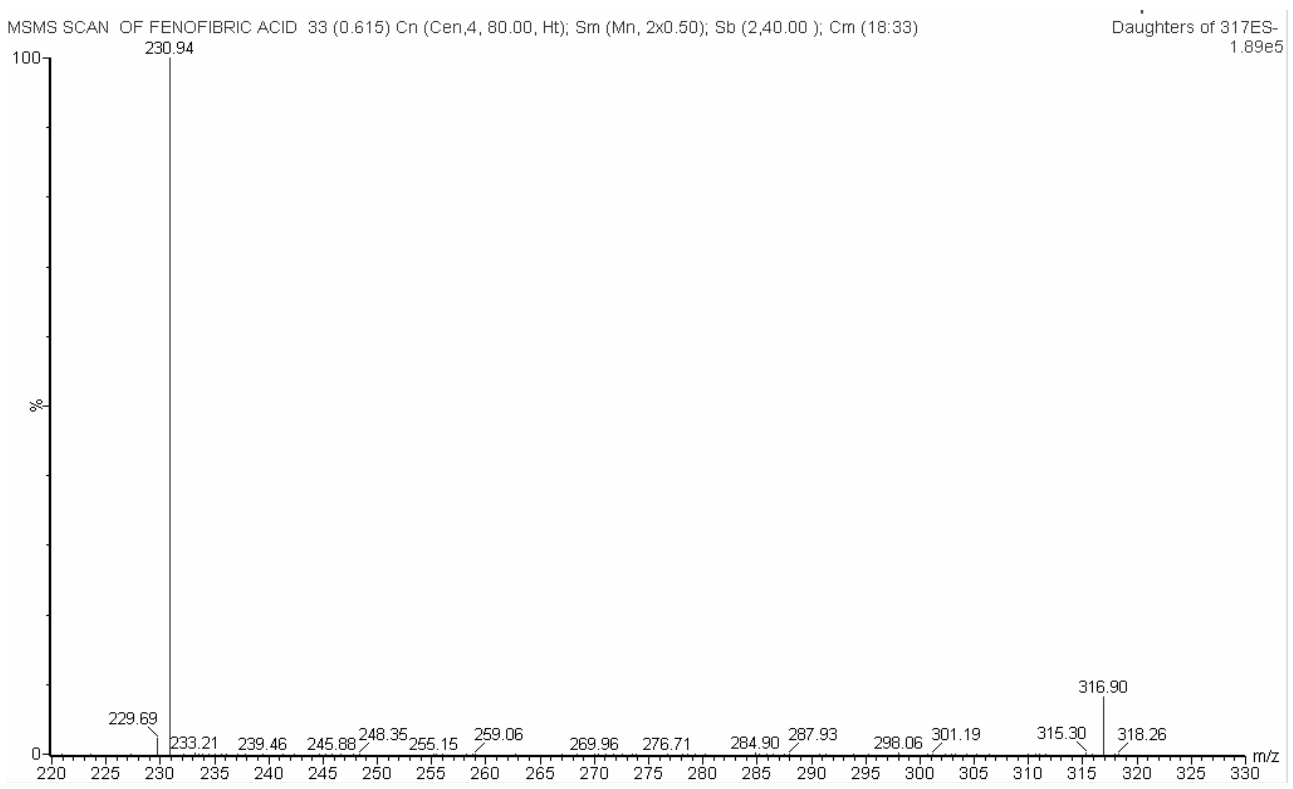

Figure 1(a). Full-scan mass spectra of fenofibric acid.

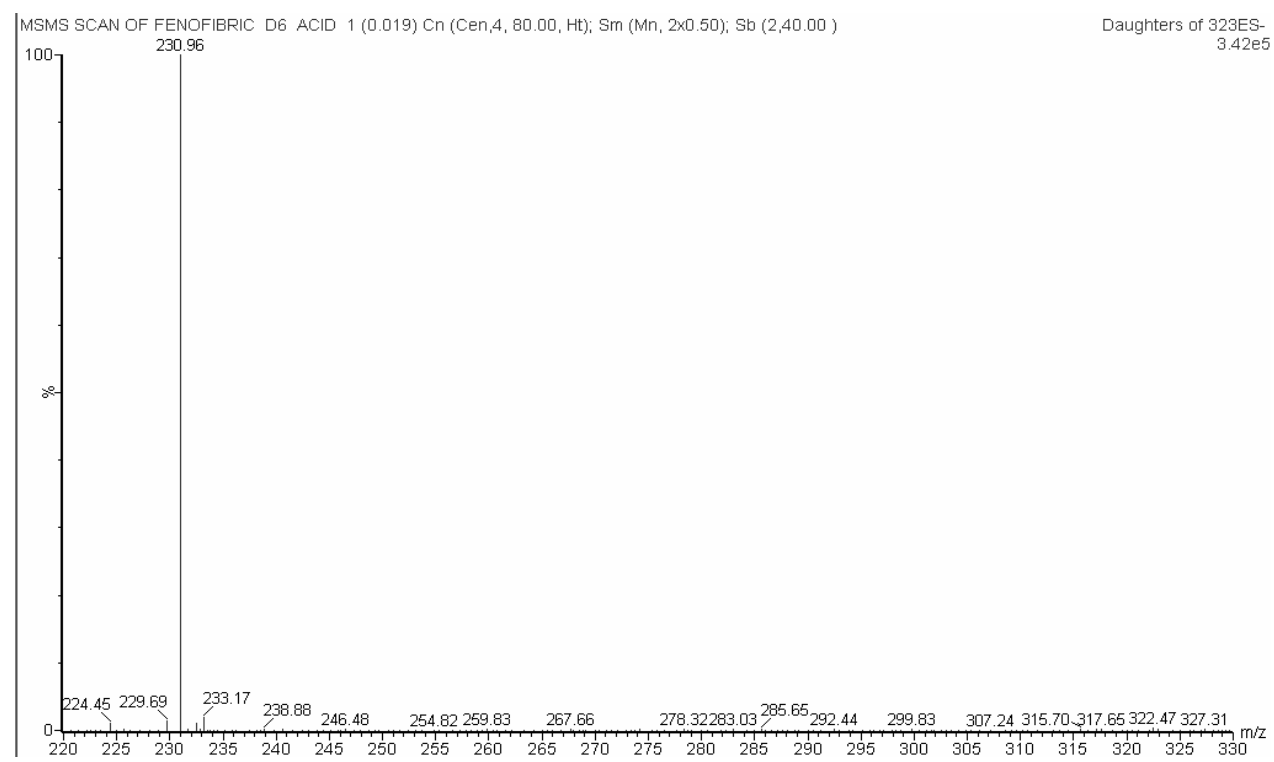

Figure 1(b). Full-scan mass spectra of fenofibric d6 acid.<smiles>CC(C)(Oc1ccc(C(=O)c2ccc(Cl)cc2)cc1)C(=O)O</smiles>

Fenofibric acid<smiles>[2H]C([2H])([2H])C(Oc1ccc(C(=O)c2ccc(Cl)cc2)cc1)(C(=O)O)C(=O)O</smiles>

Fenofibric d6 acid

Figure 2. Chemical structures of fenofibric acid and fenofibric d6 acid. 
In order to establish the optimum conditions for the SPE procedures, washing of the plasma sample before elution with methanol was done with $2 \mathrm{~mL}$ water (HPLC grade). The results indicated that proteins and interfering endogenous compounds were removed by water without any loss of the analytes. Methanol was chosen as extraction solvent for the extraction of the acidic metabolite (fenofibric acid) from the plasma which ensured the complete elution of the extracted fenofibric acid. All these procedures mentioned above ensured higher extraction recovery, better reproducibility and less interference.

\section{Assay characteristics for method validation}

\section{Selectivity}

Six lots of plasma with K3EDTA (Tri Potassium Ethylene Diamine Tetra Acetic Acid) as anticoagulant were evaluated for fenofibric acid and fenofibric d6 acid. None showed significant interfering peaks at the retention time of fenofibric acid and fenofibric d6 acid. Figure $3(a, b)$ depicts the representative chromatogram of blank sample without and with internal standard respectively.
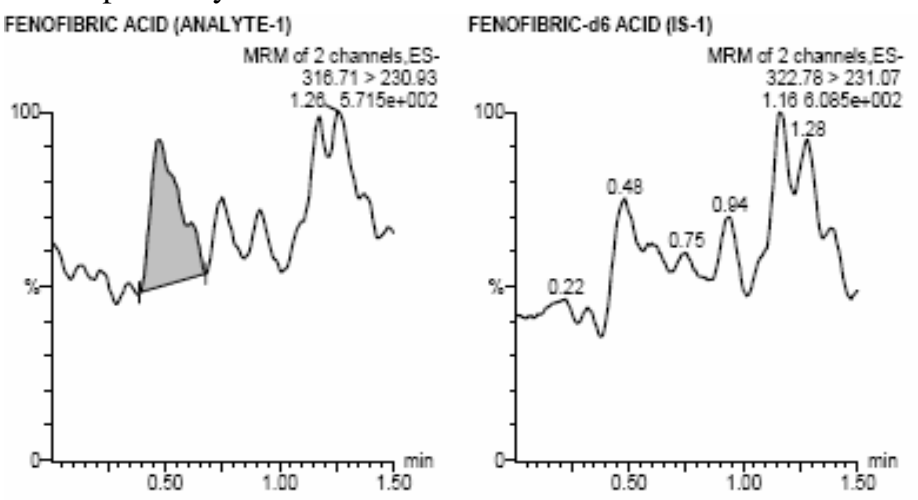

Figure 3(a). Representative chromatogram of extracted plasma blank without internal standard.
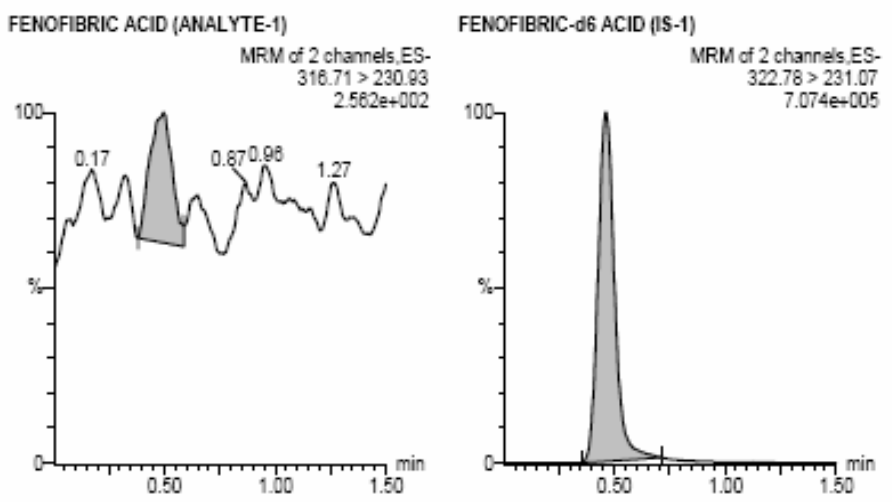

Figure 3(b). Representative chromatogram of extracted plasma blank with internal standard.

\section{Sensitivity}

The limit of quantitation was $0.176 \mu \mathrm{g} / \mathrm{mL}$ for fenofibric acid. The between batch precision and accuracy at LOQQC concentration using internal standard ratio method was $3.4 \%$ and $105.4 \%$, respectively for fenofibric acid. Figure 4 depicts the chromatogram of fenofibric acid and its internal standard from extracted plasma at LLOQ concentration. 

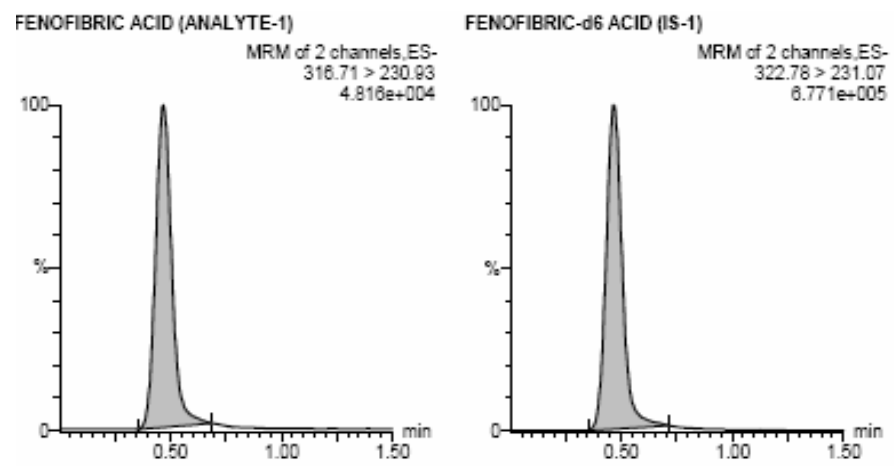

Figure 4. Representative chromatogram of fenofibric acid and its internal standard from extracted plasma at LLOQ concentration.

\section{Matrix effect}

Matrix effect is due to co-elution of some endogenous components present in biological samples. These components may not give a signal in MRM of target analyte, but can certainly decrease or increase the response of the analyte dramatically to affect the sensitivity, accuracy and precision of the method. Thus assessment of matrix effect constitutes an important and integral part of validation for quantitative LC-MS/MS method for supporting pharmacokinetic studies $^{12}$. To evaluate the matrix effect, chromatographic peak areas of fenofibric acid from the spike-after preparation samples were compared to the neat standards at the QC concentrations. The values of QCs were back calculated against freshly spiked calibration curve. At low and high quality control concentrations the precision was found to be $2.3 \% \& 3.5 \%$, respectively and accuracy was $101.9 \% \& 98.9 \%$, respectively for fenofibric acid.

\section{Linearity}

The linearity of the fenofibric acid was determined by weighted least square regression analysis of standard plot associated with nine point standard curve. The calibration was shown to be linear from $0.176 \mu \mathrm{g} / \mathrm{mL}$ to $19.837 \mu \mathrm{g} / \mathrm{mL}$. Best-fit calibration lines of chromatographic response versus concentrations were determined by weighted least square regression analysis with weighting factor of $1 /$ Concentration $^{2}$. The ' $r$ ' was consistently 0.99 or greater during the course of validation for fenofibric acid.

\section{Precision and accuracy}

The accuracy of the assay was defined as the absolute value of the calculated mean concentration of the quality control sample to their respective nominal values, expressed as percentage. Within and between batch accuracy ranged from 93.1 to $108.1 \%$ and 93.5 to 105.4 respectively for fenofibric acid (Table 1). The precision of the assay was measured by the percent coefficient of variation at concentrations of LOQQC, Low, Middle and High quality control samples. Within and between batch precision ranged from 0.5 to $4.3 \%$ and 1.6 to $3.4 \%$ respectively for fenofibric acid (Table 1).

\section{Recovery}

\section{Recovery of fenofibric acid}

The percentage recovery of fenofibric acid was determined by measuring the mean peak area response of six replicates of extracted quality control samples (LQC, MQC and HQC) against the mean peak area response of six replicates of aqueous (unextracted) quality control samples at concentrations representing $100 \%$ extraction of quality control samples at 
low, middle and high concentration. The recovery of fenofibric acid at LQC, MQC and HQC levels were $67.7,69.1$ and $72.3 \%$, respectively. The C.V. of recovery across the low, middle and high quality control concentrations was $3.4 \%$.

Table 1. Intra \& inter-batch precision \& accuracy of fenofibric acid in human plasma.

\begin{tabular}{ccccc}
\hline QC Samples & LOQQC & LQC & MQC & HQC \\
\hline Concentration, $\mu \mathrm{g} / \mathrm{mL}$ & 0.177 & 0.472 & 6.941 & 13.882 \\
Intra -batch mean & 0.1857 & 0.4817 & 6.5132 & 14.0805 \\
Intra -batch \% CV & 1.9 & 4.3 & 1.3 & 0.5 \\
Intra -batch \% SD & 0.00356 & 0.02050 & 0.08471 & 0.06643 \\
Inter -batch mean & 0.1866 & 0.4768 & 6.4873 & 13.9357 \\
Inter -batch \% CV & 3.4 & 3.3 & 1.6 & 2.6 \\
Inter -batch \% SD & 0.00633 & 0.00633 & 0.00633 & 0.00633 \\
\hline
\end{tabular}

Table 2. Precision \& accuracy of fenofibric acid in human plasma.

\begin{tabular}{lllll}
\hline QC Samples & LOQQC & LQC & MQC & HQC \\
\hline Concentration, $\mu \mathrm{g} / \mathrm{mL}$ & 0.177 & 0.472 & 6.941 & 13.882 \\
mean & 0.1945 & 0.4675 & 6.5227 & 13.4180 \\
$\% \mathrm{CV}$ & 2.4 & 0.8 & 3.3 & 2.2 \\
$\%$ Nominal & 109.9 & 99.0 & 94.0 & 96.7 \\
\hline
\end{tabular}

Recovery of fenofibric $d 6$ acid

The percentage recovery of fenofibric $\mathrm{d} 6$ acid was determined by measuring the mean peak area response of fenofibric d6 acid in extracted quality control samples (MQC) against the mean peak area response of aqueous (unextracted) internal standards solution at concentration representing $100 \%$ extraction of internal standards in MQC samples. The recovery of fenofibric $\mathrm{d} 6$ acid was $88.8 \%$.

\section{Stability}

Freeze-thaw stability

The stability of spiked plasma samples was determined after three freeze thaw cycles. Six replicate of low and high quality control samples were analysed after third freeze thaw cycle. The percentage degradation was determined by calculating the concentration of stability quality control samples against freshly spiked calibration curve. The stability and precision ranged from 101.1 to $102.5 \%$ and 1.5 to $1.6 \%$, respectively for fenofibric acid (Table 3).

\section{Bench- top stability}

The bench top stability evaluation involved analysis of six replicates of low and high QC stability samples, which had been kept at room temperature for a period of $7.60 \mathrm{~h}$ against freshly spiked calibration curve. The stability and precision ranged from $101.6 \%$ to $103.0 \%$ and 1.4 to $1.7 \%$, respectively for fenofibric acid (Table 3 ).

\section{In-injector stability}

In-injector stability of six replicate quality control samples was determined. Low and high QC samples kept in auto-injector were analyzed after $49.87 \mathrm{~h}$ and the concentrations were calculated against freshly spiked calibration curve. The stability and precision ranged from 98.6 to $100.1 \%$ and 1.2 to $1.6 \%$, respectively for fenofibric acid (Table 3 ). 


\section{Post preparative stability}

Post preparative stability of six replicate quality control samples was determined for the time period for which processed QC Samples were kept in refrigerator. Processed low and high QC samples were kept in refrigerator for $24.35 \mathrm{~h}$ and the concentrations were calculated against freshly spiked calibration curve. The stability and precision ranged from 102.4 to $105.6 \%$ and 0.7 to $1.6 \%$, respectively for fenofibric acid (Table 3).

Table 3. Stability of fenofibric acid in human plasma

\begin{tabular}{|c|c|c|c|}
\hline Parameters & QC Samples & LQC & HQC \\
\hline \multirow{4}{*}{$\begin{array}{l}\text { Bench Top Stability } \\
\text { (for } 7.60 \mathrm{~h} \text { ) }\end{array}$} & $\begin{array}{c}\text { Concentration, } \\
\mu \mathrm{g} / \mathrm{mL}\end{array}$ & 0.177 & 6.941 \\
\hline & mean & 0.4860 & 14.1102 \\
\hline & $\% \mathrm{CV}$ & 1.7 & 1.4 \\
\hline & $\%$ Nominal & 103.0 & 101.6 \\
\hline \multirow{3}{*}{$\begin{array}{l}\text { Freeze Thaw Stability } \\
\text { (for three cycles) }\end{array}$} & mean & 0.4840 & 14.0365 \\
\hline & $\% \mathrm{CV}$ & 1.5 & 1.6 \\
\hline & $\%$ Nominal & 102.5 & 101.1 \\
\hline \multirow{3}{*}{$\begin{array}{l}\text { In-Injector Stability } \\
\text { (for } 49.87 \mathrm{~h} \text { ) }\end{array}$} & mean & 0.4725 & 13.6913 \\
\hline & $\% \mathrm{CV}$ & 1.2 & 1.6 \\
\hline & $\%$ Nominal & 100.1 & 98.6 \\
\hline $\begin{array}{l}\text { Long Term Stability } \\
\left(\text { below }-15{ }^{\circ} \mathrm{C} \text { ) }\right.\end{array}$ & $\begin{array}{c}\text { mean } \\
\% \mathrm{CV} \\
\% \text { Nominal }\end{array}$ & $\begin{array}{c}0.4825 \\
1.3 \\
102.2\end{array}$ & $\begin{array}{c}14.4388 \\
1.4 \\
104.0\end{array}$ \\
\hline $\begin{array}{l}\text { Long Term Stability } \\
\left(\text { below }-50{ }^{\circ} \mathrm{C} \text { ) }\right.\end{array}$ & $\begin{array}{c}\text { mean } \\
\% \mathrm{CV} \\
\% \text { Nominal }\end{array}$ & $\begin{array}{c}0.4815 \\
1.7 \\
102.0\end{array}$ & $\begin{array}{c}14.0160 \\
1.4 \\
101.0\end{array}$ \\
\hline Post Processing Refrigerator & mean & 0.4833 & 14.6598 \\
\hline Stability & $\% \mathrm{CV}$ & 1.6 & 0.7 \\
\hline (for $24.35 \mathrm{~h}$ ) & $\%$ Nominal & 102.4 & 105.6 \\
\hline
\end{tabular}

\section{Stock Solution Stability of fenofibric acid and fenofibric $d 6$ acid}

The stock solutions of fenofibric acid and fenofibric d6 acid (both protected from light) were found to be stable for 14 days and 15 days, respectively when stored at refrigerated temperature (between $1-10^{\circ} \mathrm{C}$ ). The percentage stability of the stock solutions for fenofibric acid and fenofibric d6 acid were 108.3 and $95.2 \%$, respectively. The mean responses of stability samples were within the acceptance criteria of $85-115 \%$ of the mean response of comparison samples.

\section{Short term stability of fenofibric acid and fenofibric $d 6$ acid}

Short-term stability for fenofibric acid and fenofibric d6 acid (both protected from light) were determined. The stability stock dilutions for fenofibric acid stored at room temperature for 9.65 hours and the stability stock dilutions for fenofibric d6 acid stored at room temperature for 9.60 hours were analyzed against freshly prepared stock dilutions (comparison stock dilutions) from the same standard stock solution used for preparing the stability dilutions. The stability of the stock dilutions for fenofibric acid and fenofibric $\mathrm{d} 6$ acid were 99.1 and $98.7 \%$, respectively. The precision was $0.5 \%$ for stability and $0.7 \%$ for comparison dilutions for fenofibric acid. The precision was $0.7 \%$ for stability and $0.8 \%$ for comparison dilutions for fenofibric $\mathrm{d} 6$ acid. 


\section{Short term stability of reference solution}

Short-term stability of reference solution of fenofibric acid stored at room temperature (protected from light) was determined. The stability reference solution dilutions stored at room temperature for 9.70 hours were analyzed against freshly prepared reference solution dilutions (comparison dilutions). The stability of fenofibric acid in reference solution was $99.9 \%$.

\section{Long term stability}

Long-term stability of fenofibric acid was evaluated using low and high QC samples stored below $-15{ }^{\circ} \mathrm{C}$ in cold room and below $-50{ }^{\circ} \mathrm{C}$ in deep freezer for a period of 102 days. Six replicates of low and high quality control samples were used for each stability exercise. The stored QC samples were analyzed against freshly spiked calibration curve (Table 3).

\section{Dilution integrity}

Dilution integrity was determined by spiking about 1.79 times ULOQ concentration for fenofibric acid. This was then diluted by factor of 2 and 4 with drug free plasma.The accuracy for two times diluted concentration was $97.6 \%$ and four times diluted concentration was $99.4 \%$ and precision for two times diluted concentration was $3.3 \%$ and four times diluted concentration was $2.6 \%$ for fenofibric acid

\section{Application of the method in pharmacokinetic studies}

The established LC-MS/MS method was successfully applied to the determination of fenofibric acid in the plasma samples after oral administration of $145 \mathrm{mg}$ fenofibrate in healthy Indian volunteers. The mean plasma concentration-time profiles are shown in Figure 5. Pharmacokinetic parameters for fenofibrate test and reference formulation are shown in Table 4.

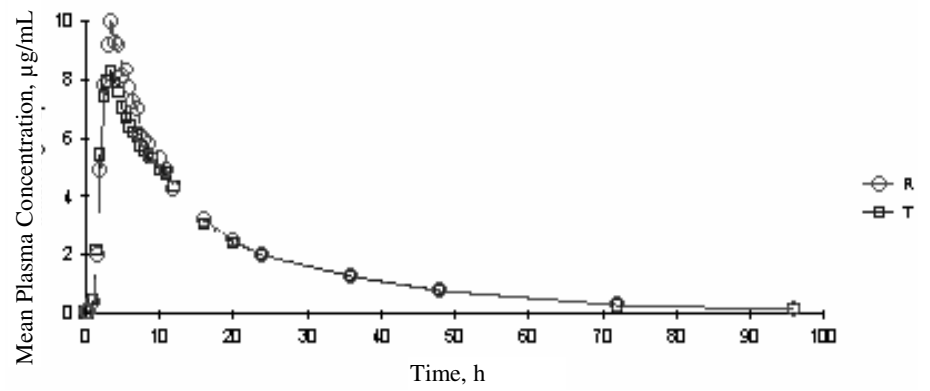

Figure 5. Linear mean plasma concentration-time profile for fenofibric acid fed study (145 mg tablet)

Table 4. Summary results of pharmacokinetic parameters for fenofibric acid.

\begin{tabular}{lcc}
\hline \multicolumn{1}{c}{ QC Samples } & Reference & Test \\
\hline $\mathrm{T}_{\max }, \mathrm{h}$ & $3.800 \pm 1.2867$ & $3.400 \pm 0.9189$ \\
$\mathrm{C}_{\max }, \mu \mathrm{g} / \mathrm{mL}$ & $10.3908 \pm 2.13160$ & $9.1142 \pm 2.01177$ \\
$\mathrm{AUC}_{0-\mathrm{t}}, \mathrm{h} \mathrm{ug} / \mathrm{mL}$ & $151.5652 \pm 68.03542$ & $141.0268 \pm 64.49053$ \\
$\mathrm{AUC}_{0-\infty}, \mathrm{h} \mathrm{ug} / \mathrm{mL}$ & $158.2230 \pm 69.81120$ & $151.4068 \pm 69.51367$ \\
\hline
\end{tabular}

\section{Conclusion}

This method had a suitable LOQ of $0.176 \mu \mathrm{g} / \mathrm{mL}$ along with the very short retention time of 1.5 minute was useful for analyzing more than 900 samples in a single day which is the most advantageous aspect in comparison to other analysis techniques available in published literature. In addition to this, a high recovery of both fenofibric acid (65-73\%) as well as fenofibric $\mathrm{d} 6$ acid $(88.8 \%)$ was obtained. Both fenofibric acid and fenofibric d6 acid were stable below 
$-15{ }^{\circ} \mathrm{C}$ as well as $-50{ }^{\circ} \mathrm{C}$ for 102 days. This method employs a very simple cost effective solid phase extraction procedure finally requiring very less injection volume $(5 \mu \mathrm{L})$ resulting in high throughput bioanalysis technique. The method had advantage over problems of poor chromatography, questionable uncharacterized peak, tedious extraction steps, high injection load. The method is fully validated as per USFDA guidelines, for the first time meets these challenges. Our research thus confidently stands for the first time as a simple, robust and high throughput method requiring less than 1.5 minutes to run each sample. Thus, as the pace of drug development quickens, we cannot ignore the potential for our method application nor the time savings it can present thus encouraging clinical and exploratory studies for the best possible lipid regulating agents. The established LC-MS/MS method was successfully applied to the determination of fenofibric acid in the plasma samples after oral administration of $145 \mathrm{mg}$ fenofibric acid in 10 healthy Indian volunteers.

\section{Acknowledgement}

The authors would like to thank Ranbaxy Laboratories Ltd. for giving permission to write this paper and to use in-house data.

\section{References}

1. $\quad$ Edgar A D, Curr Ther Res Clin Exp., 1990, 47, 952-961.

2. Knopp R H, Walden C E and Warnick G R, Am J Med., 1987, 83, 75-84.

3. Guay D R, Ann Pharmacother., 1999, 33, 1083-1103.

4. Balfour J A, McTavish D and Heel R C, Drugs, 1990, 40, 260-290.

5. Trivedi R K, Kallem R R, Mullangi R and Srinivas N R, J Pharm Biomed Anal., 2005 39(3-4), 661-9.

6. Mertens B, Cahay B, Klinkenberg R and Streel B, J Chromatogr., 2008, 1189, 493-502.

7. Guidance for Industry: Bioavailability and Bioequivalence Studies for Orally Administered Drug Products-General Considerations, 2003.

8. Method Validation for Extemporaneous Pediatric Formulation Prototypes of Quinapril Hydrochloride/ Assay and Stability Method. Pfizer Global Research and Development, Ann Arbor, Michigan, November, 2001.

9. Validation of Analytical Procedures: Methodology, ICH-Q2B, International Conference on Harmonization of Technical Requirements for the Registration of Pharmaceuticals for Human Use, Geneva, Switzerland, 1996.

10. Guidance for Industry: Analytical Procedures and Method Validation, U.S. Food and Drug Administration, Center for Drug Evaluation and Research, Rockville, Maryland, 2000.

11. Validation of Analytical Methods for Drug Products, Pfizer Global Research and Development, Ann Arbor, Michigan, 2001.

12. Parekh S A., Pudage A, J Chromatogr B., 2008, 867, 178.

13. Dams R, Huestis M A, Lambert W E and Murphy C M, J Am Soc Mass Spectrom., 2003, 14, 1290.

14. L Jia, Young X and Guo W, J Pharm Sci., 1999, 88(10), 981.

15. L Jia, Wong H, Wang Y and Garza M., J Pharm Sci., 2003, 92, 161. 


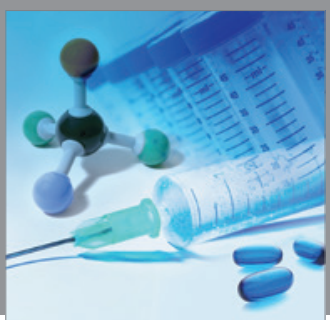

International Journal of

Medicinal Chemistry

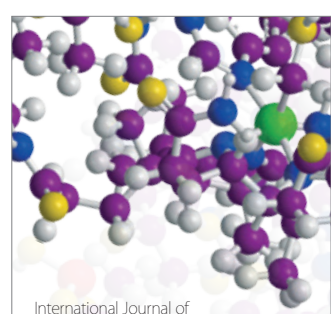

Carbohydrate Chemistry

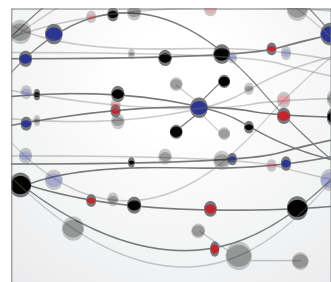

The Scientific World Journal
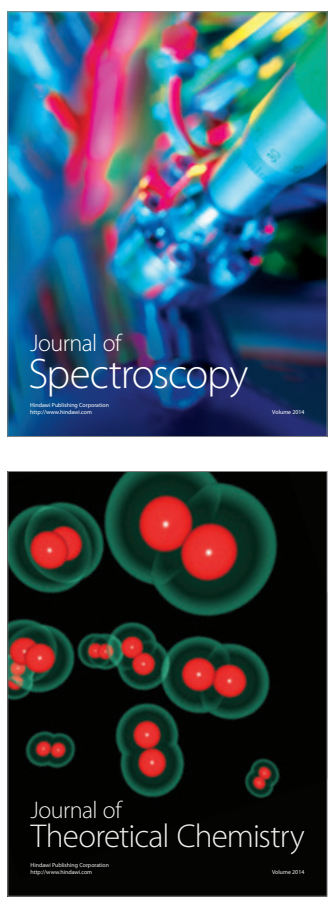
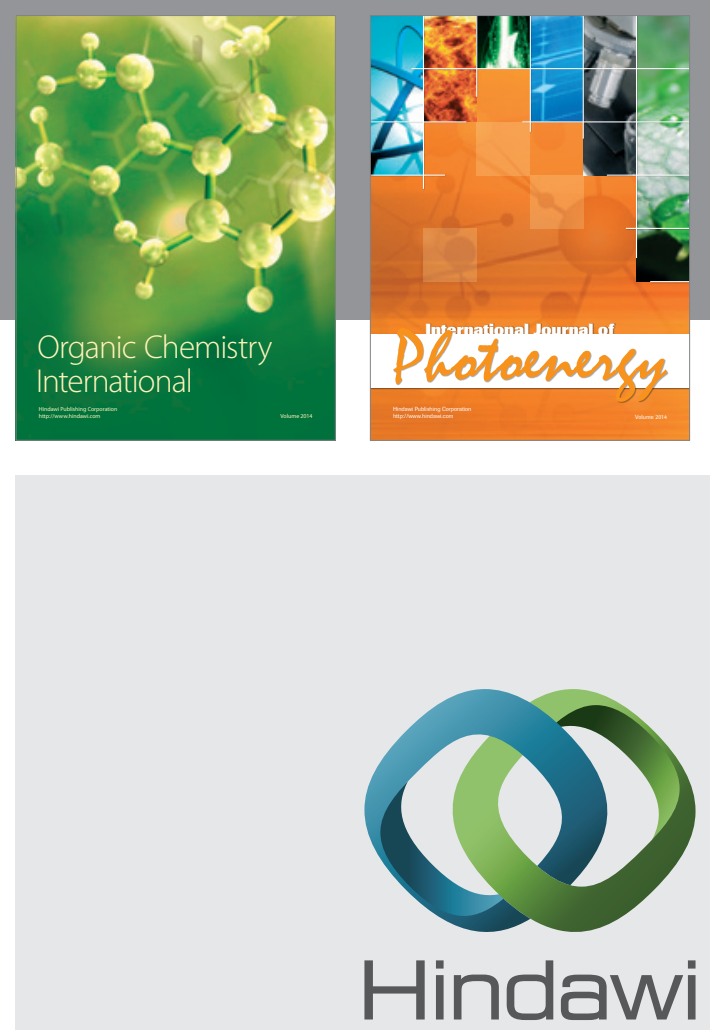

Submit your manuscripts at

http://www.hindawi.com
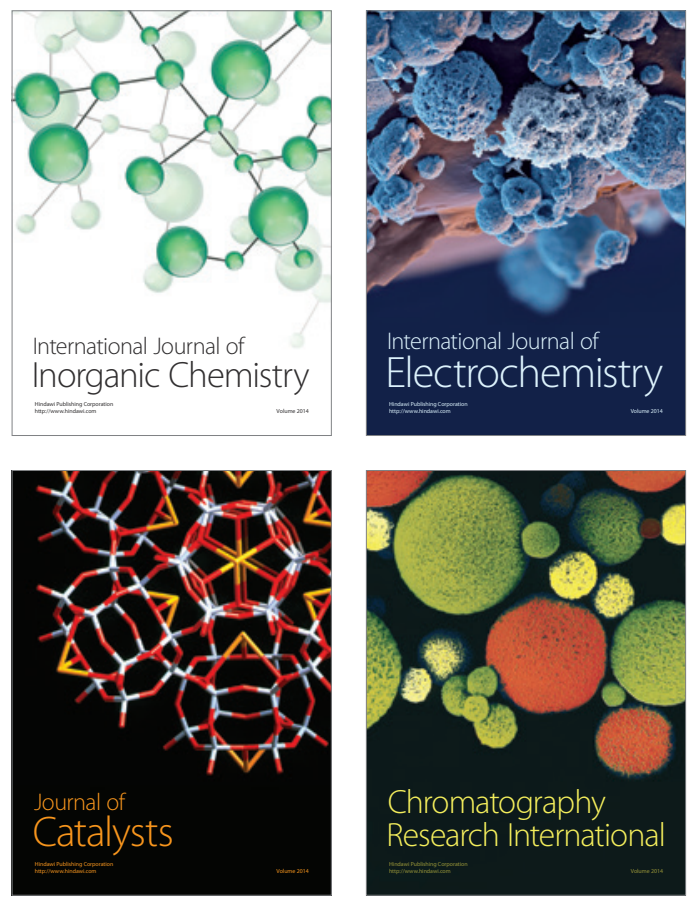
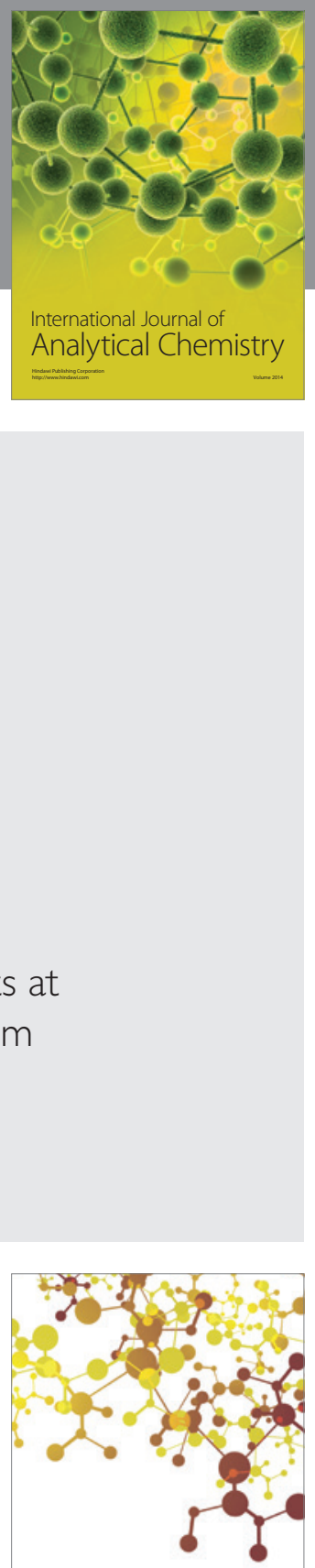

Journal of

Applied Chemistry
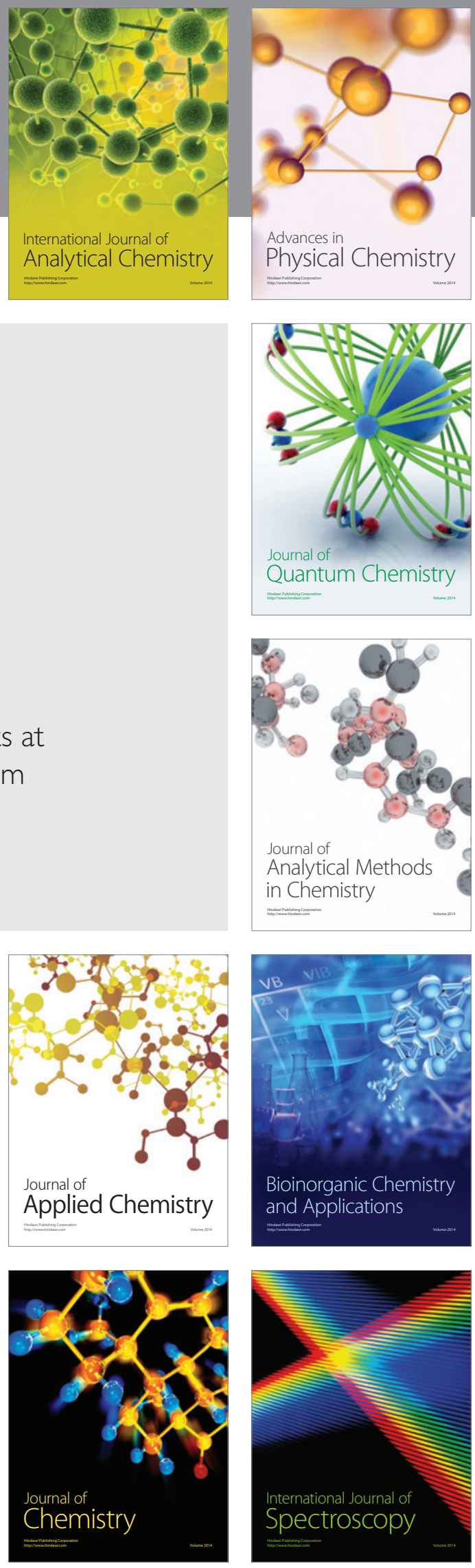